\title{
Prospecção tecnológica: o avanço dos estudos de caprinos
}

\author{
Technological prospecting: the advancement of goat studies \\ Prospección tecnológica: el avance de los estudios caprinos
}

Recebido: 20/09/2021 | Revisado: 29/09/2021 | Aceito: 01/10/2021 | Publicado: 04/10/2021

\author{
Ariadne Soares Meira \\ ORCID: https://orcid.org/0000-0001-8740-9009 \\ Universidade Federal de Campina Grande, Brasil \\ E-mail: ariadnesm_eng@hotmail.com \\ Diego José Araújo Bandeira \\ ORCID: https://orcid.org/0000-0002-6245-9875 \\ Universidade Federal de Campina Grande, Brasil \\ E-mail: diegoimperium8@gmail.com \\ Antônio Nunes de Oliveira \\ ORCID: https://orcid.org/0000-0001-5697-8110 \\ Instituto Federal do Ceará, Brasil \\ E-mail: nunes.vieira@ifce.edu.br \\ Anúbes Pereira de Castro \\ ORCID: https://orcid.org/0000-0002-3795-5666 \\ Universidade Federal de Campina Grande, Brasil \\ E-mail: anubescastro@gmail.com \\ Patrício Gomes Leite \\ ORCID: https://orcid.org/0000-0001-5500-7865 \\ Universidade Federal do Maranhão, Brasil \\ E-mail: pgomesleite@gmail.com \\ Kaline Lígia do Nascimento \\ ORCID: https://orcid.org/0000-0002-9831-7166 \\ Universidade Estadual da Paraíba, Brasil \\ E-mail:kaline.dmi@hotmail.com \\ Keviane Pereira Aragão \\ ORCID: https://orcid.org/0000-0002-2237-130X \\ Universidade Federal de Campina Grande, Brasil \\ E-mail: kevianearagao@gmail.com \\ Marcia Janiele Nunes da Cunha Lima \\ ORCID: https://orcid.org/0000-0003-3697-8724 \\ Universidade Federal de Campina Grande, Brasil \\ E-mail: marciacunhalima87@hotmail.com
}

\section{Resumo}

A prospecção tecnológica é um processo de inteligência competitiva que permite rastrear a informação disponível em todo o mundo e disponibiliza-la aos pesquisadores e/ou às empresas parceiras oferecendo panoramas de linhas de investigação que podem seguir e encontrar novos nichos de mercado. Demanda cuidado e especificações aliados à pesquisa, incutindo a inovação nos métodos e processos de gestão frente às exigências de um mercado globalizado, com concorrência acirrada, elevado grau de incertezas e um grande volume de informação disponível. Partindo desse pressuposto, este trabalho teve como objetivo a busca por pedidos de patentes, patentes já concedidas e modelo de utili dade evidenciando a palavra "caprinos" para que fosse possível a quantificação, classificação e destinação desses pedidos. Para isso foi necessário uma busca no site do INPI (Instituto Nacional de Propriedade Industrial) para o conhecimento dos registros, assim como no Google Patents e Espacenet, sendo encontrados 137 arquivos referentes ao tema em busca. Após isso, realizou-se uma seleção e classificação deste material para que fosse possível observar as finalidades específicas destes pedidos e assim, se chegar a uma assertiva no que concerne a finalidade dos arquivos analisados.

Palavras-chave: Depósito; Patentes; Propriedade intelectual.

\begin{abstract}
The technological prospection is competitive intelligence processes that allows you to track the information available worldwide and provides it to researchers and/or companies offering panoramas of lines of inquiry that can follow and find new niche markets. Demand care and allied research specifications, instilling the innovation in management methods and processes against the demands of a globalized market, with tough competition, high degree of uncertainty and a large volume of information available. Starting from this assumption, this work had as objective the search for patent applications, patents already granted and utility model showing the word "goats" to quantification,
\end{abstract}


classification and allocation of these requests. This required a search on the website of the INPI (National Institute of Industrial Property) to the knowledge of the records as well as on Google Patents and Espacenet, being found 137 search theme-related files. After that, a selection and classification of this material so that it was possible to observe the specific purposes of these requests and thus achieve an assertive regarding the purpose of the files examined.

Keywords: Deposit; Patents; Intellectual property.

\section{Resumen}

La prospección tecnológica es un proceso de inteligencia competitiva que permite realizar un seguimiento de la información disponible en todo el mundo y ponerla a disposición de investigadores y/o empresas asociadas ofreciendo panoramas de líneas de investigación que pueden seguir y encontrar nuevos nichos de mercado. Cuidado de la demanda y especificaciones aliadas a la investigación, inculcando la innovación en los métodos y procesos de gestión frente a las demandas de un mercado globalizado, con una competencia feroz, alto grado de incertidumbre y un gran volumen de información disponible. Partiendo de este supuesto, este trabajo tuvo como objetivo buscar solicitudes de patentes, patentes ya concedidas y modelo de utilidad que evidence la palabra "cabras" para que fuera posible cuantificar, clasificar y asignar estas solicitudes. Para ello fue necesaria una búsqueda en el sitio del INPI (Instituto Nacional de la Propiedad Industrial) para el conocimiento de los registros, así como en Google Patentes y Espacenet, encontrándose 137 archivos relacionados con el tema en búsqueda. Después de eso, se realizó una selección y clasificación de este material para que fuera posible observar los propósitos específicos de estas solicitudes y, por lo tanto, si se trata de una afirmación con respecto al propósito de los archivos analizados.

Palabras clave: Presentación; Patentes; Propiedad intelectual.

\section{Introdução}

Segundo Medeiros (2016); Barros e Cruz (2017) e Oliveira (2020), a criação de caprinos, seja para abate, elite ou produção de leite, destaca-se pela sua produtividade e por sua adaptabilidade e como em outros lugares é uma das atividades mais comuns no nordeste brasileiro, principalmente no cariri. O caprino encontrou nessa região condições satisfatórias para desempenhar suas funções fisiológicas com total potencial, ou seja, condições favoráveis a sua condição de vida e sua procriação. Por ser um animal com fortes raízes com seus antepassados selvagens, demandam técnicas de manejo adequadas para que possam cumprir o fim a qual estão destinados.

De acordo com uma pesquisa do Instituto Brasileiro de Geografia e Estatística - IBGE, o rebanho nacional de caprinos em 2016 alcançou 8.851 .879 cabeças, sendo 8.109.672 cabeças na Região Nordeste (91,6\%), enquanto o rebanho ovino registrou em 2014 o número de 17.614.454 cabeças no País, das quais 10.126 .799 estão no Nordeste (57,5\%) e 5.166.225 na Região Sul (29,3\%). Em termos de tendência nota-se uma diminuição do rebanho na série de 2005 a 2014 , para o rebanho caprino, diferentemente do que se observa para o ovino.

Tendo em vista o manejo do rebanho de caprinos, Barbosa Filho (2013), Campos et al. (2017) e Cruz et al. (2019) especificam que diversos acessórios e ferramentas foram desenvolvidos para caprinocultura, seja para facilitar o manejo, abate, processamento e até mesmo a ingestão dos alimentos oriundos desta atividade. Por consequência os registros indicam as especificidades do invento e garantem a quem inventou o direito de usufruir financeiramente de sua criação.

O termo prospecção tecnológico designa atividades de prospecção centrada nas mudanças tecnológicas, em mudanças na capacidade funcional ou no tempo e significado de uma inovação, tendo por objetivo o delineamento e testes das visões possíveis e desejáveis para que sejam feitas escolhas que contribuirão de forma significativa para a produção (AMPARO, 2012).

Com isto, o presente trabalho teve por objetivo a busca por pedidos de patentes, patentes concedidas e modelo de utilidade, evidenciando a palavra "caprinos" para que fosse possível a quantificação, classificação e destinação desses pedidos nos sites do INPI (Instituto Nacional de Propriedade Industrial), assim como no Google Patentes e Espacenet.

\section{Metodologia}

Foram realizadas pesquisas utilizando palavras chaves para caprino no INPI, Google Patents e Espacenet, utilizando 
como dados de entrada as palavras "caprinos" no plural, "caprino" no singular, as palavras em inglês "goats e goat" as quais remetem a caprinos e caprino e uma variação da palavra também para caprino na língua inglesa, "caprine” (Tabela 1).

Os dados coletados foram para o ano de referência de 2016, sendo realizada uma avaliação cronológica desde o ano de 1991 até o ano de 2015.

Tabela 1. Prospecção para a palavra "caprinos" e suas variações.

\begin{tabular}{cccc}
\hline Palavras-chave & INPI & Google Patents & Espacenet \\
Caprinos & 85 & 95 & 0 \\
Caprino & 12 & 400 & 5 \\
Goats & 0 & 111.000 & 221.000 \\
Goat & 0 & 10.300 & 3.808 \\
Caprine & 0 & 115 \\
\hline
\end{tabular}

Fonte: Autores.

Foi dado ênfase aos processos de deposito de patentes encontrados no INPI tendo em vista que a pesquisa realizada nos sites de patentes internacionais detém uma quantidade muito elevada de patentes.

No site do INPI foram realizadas pesquisas avançadas inserindo a palavra chave "caprino" no singular e no plural para ser encontrada tanto no título quanto no resumo.

No total foram encontrados 137 (cento e trinta e seis) processos no banco de dados do INPI onde noventa e seis foram deposito em PI (Patente de Invenção) e vinte depósitos em UM (Modelo de Utilidade), sendo encontrados agentes depositantes oriundos do Brasil assim como de países como a Holanda, Portugal, México, Itália e Estados Unidos.

\section{Resultados e Discussão}

Ao ser inserida a palavra "caprino" no título foram encontrados cinco processos. Quando inserida no título a palavra “caprinos", no plural, foram encontrados trinta e cinco processos sendo depositados todos por agentes no Brasil. Ao serem submetidos, vinte e sete destes foram identificados como PI e oito identificados como MU.

Ao ser inserida no resumo a palavra "caprino", foram encontrados doze processos sendo depositados nove por agentes no Brasil e três por agentes na Holanda. Ao serem submetidos, onze destes foram identificados como PI e apenas um identificado como MU.

Quando inserida no resumo a palavra "caprinos", no plural, foram encontrados oitenta e cinco processos sendo depositados todos por oitenta e um agentes no Brasil e quatro agentes em países distintos. Ao serem submetidos, cinquenta e três destes foram identificados como PI, onze destes identificados como UM (Modelo de Utilidade), dois identificados como C1 (certificação de Adição) e um como PP (Pedido de Patente). Ainda foram encontrados dezoito com a indicação BR no início de sua classificação, indicando patentes legitimamente brasileiras (Figura 1). 
Figura 1. Número de patentes depositadas nas mais diversas categorias.

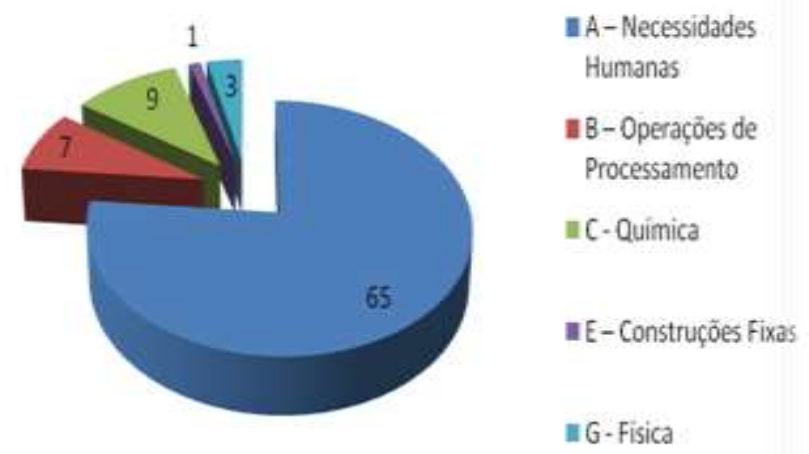

Fonte: Autores.

Autores como Silva et al. (2019) e Feitosa et al. (2021), ao também realizarem prospecção tecnológica utilizando o banco de patentes brasileiro INPI, e entrando com termos "caprinos" e "ovinos", encontraram mais de 250 respostas para as mais diversas utilizações e variações de produtos derivados destes animais.

A evolução do depósito de patentes contendo o termo "caprinos" não apresenta linearidade haja vista que a criação destes animais vem de longa data podendo ser evidenciados os anos entre 2001 e 2010 com uma quantidade maior de depósitos (Figura 2).

Figura 2. Histórico de deposito de patentes com o termo “caprinos" entre os anos de 1991 e 2015.

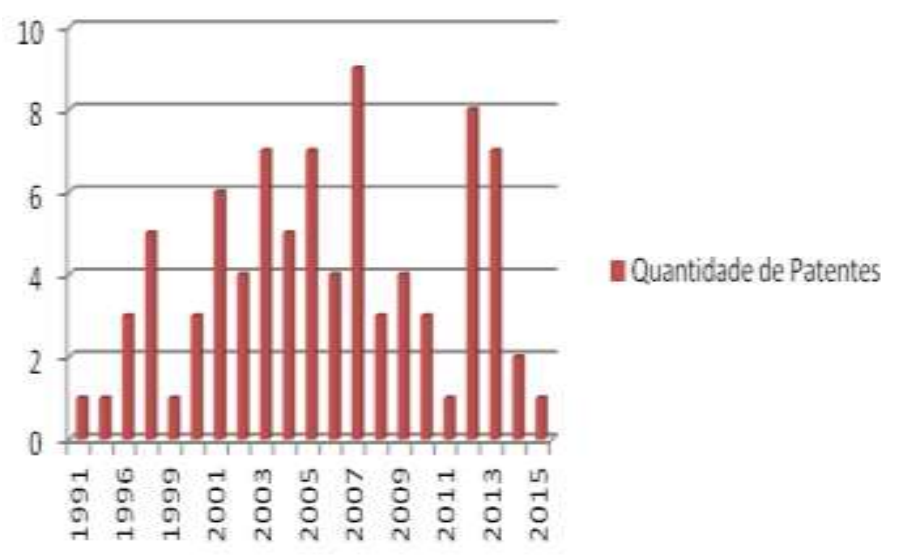

Fonte: Autores.

Conforme Vaz (2019) o Brasil apresentou um crescimento substantivo na ordem de 48\% no número de depósito de patentes entre os anos de 2000 e 2016 mesmo havendo um retrocesso de aproximadamente $7 \%$ entre os anos de 2016 e 2019.

Com relação aos depósitos relacionados a caprinos, o decréscimo no número de depósitos no ano de 2011 pode ser relacionado ao início da seca que acometeu o Nordeste do Brasil. Já nos anos posteriores de 2013 e 2014 o aumento da quantidade de depósitos, também pode estar relacionada à seca, porém, com intervenções para melhor manejo dos animais e artifícios para adaptação a esta adversidade climática.

Além do Brasil, há deposito de patentes de agentes da Holanda, Portugal, México, Itália e Estados Unidos, sendo o 
Brasil de maior expressão numérica (Figura 3).

Figura 3. Países com deposito de patente no INPI.

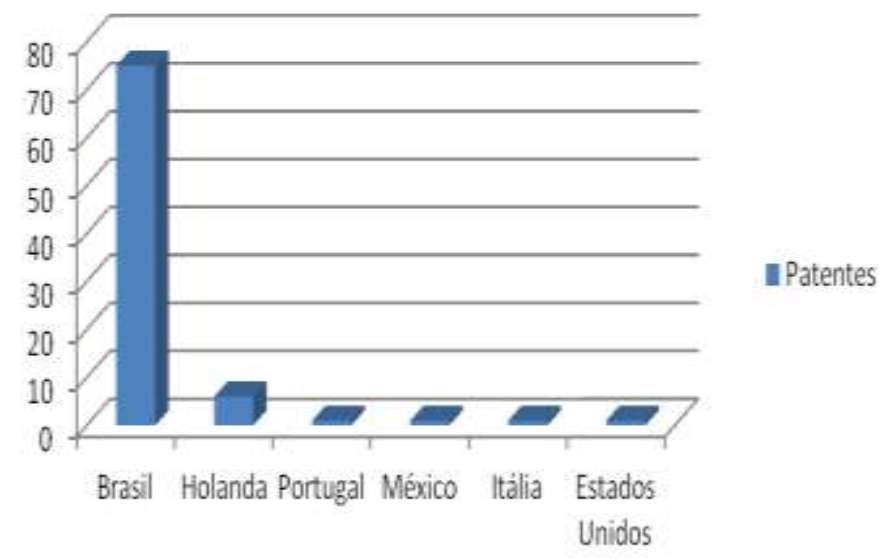

Fonte: Autores.

Por se grande criatório de caprinos e pelo fato de o INPI ser um instituto brasileiro, já era esperado que o número de depósitos de agentes brasileiros fosse superior aos depósitos dos agentes de outras nacionalidades.

Destaca-se que, os depósitos da Holanda e Itália são a respeito de tratamentos químicos aplicados ao couro advindo dos caprinos, assim como, confecção de medicamento para tratamento de enfermidades destes animais.

No Brasil, o estado de São Paulo destaca-se no depósito de patentes sobre "caprinos” mesmo sabendo-se que, o maior detentor do rebanho capril do Brasil é a região Nordeste (Figura 4).

Figura 4. Estados brasileiros depositantes.

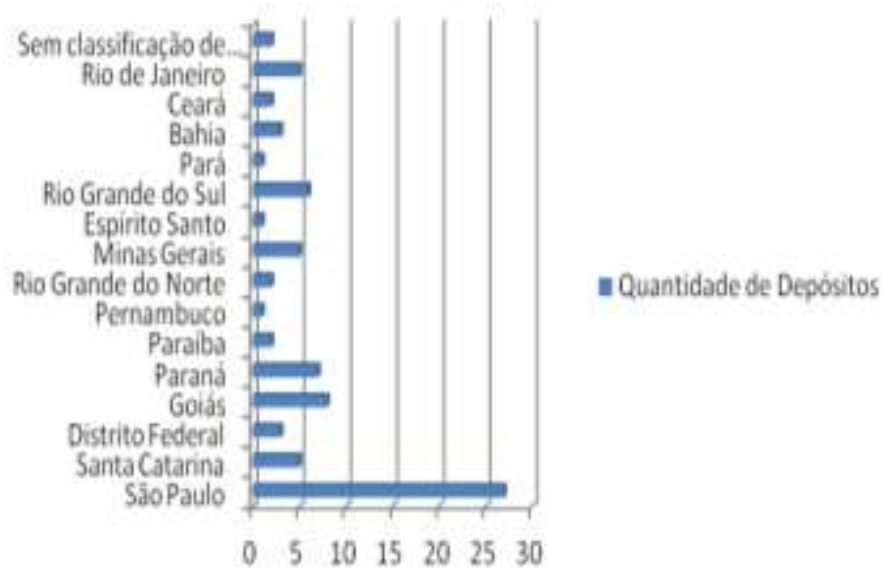

Fonte: Autores.

Entre os estados da Paraíba, Pernambuco, Rio Grande do Norte, Ceará e Bahia só existem, ao todo, o depósito de 10 patentes com o termo "caprinos", evidenciando que o manejo deste tipo de animal é escasso de tecnificação, ou a falta de interesse de pesquisadores em patentear. Conforme Silva (2019) pelo fato da caprinocultura ter muita força na região nordeste do Brasil, faz-se necessário deposito de patente de produtos destinados ao manejo dos animais ou a confecção de produtos a partir destes. 
Os maiores valores de depósitos estão relacionados com preparações medicinais contendo ingredientes ativos orgânicos, voltados para o bem-estar animal (Figura 5).

Figura 5. Quantidades de pedidos com base A61K.

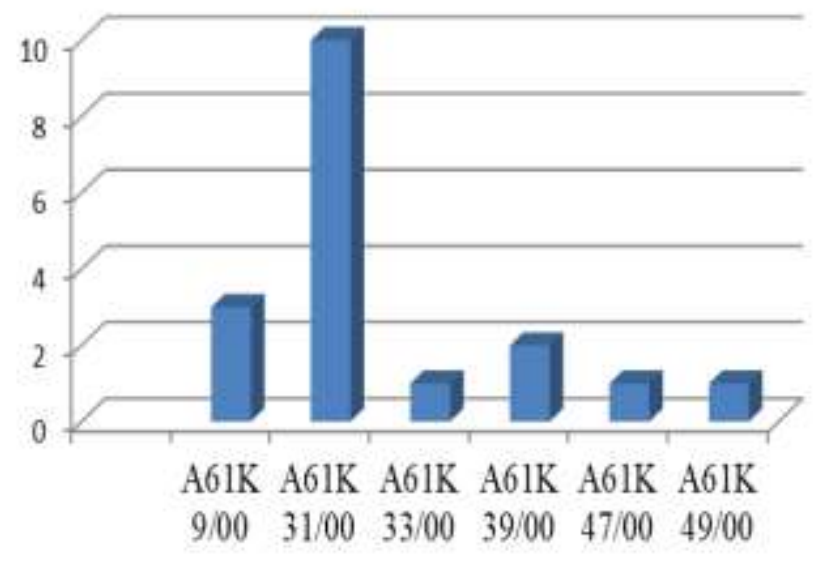

Fonte: Autores.

Dentro do banco de patentes, são designadas classificações de acordo com a finalidade da invenção. Para "caprinos" a classificação com relação a preparações medicinais é a seguinte: A61K 9/00 - Preparações medicinais caracterizadas por formas físicas especiais; A61K 31/00 - Preparações medicinais contendo ingredientes ativos orgânicos; A61K 33/00 Preparações medicinais contendo substâncias ativas inorgânicas; A61K 39/00 - Preparações medicinais contendo antígenos ou anticorpos; A61K 47/00 - Preparações medicinais caracterizadas pelos ingredientes não ativos usados, p. ex. excipientes, aditivos inertes e A61K 49/00 - Preparações para testes in vivo.

Observamos os depósitos relacionados com preparações medicinais contendo ingredientes ativos orgânicos, voltados para o bem-estar com relação à classificação da patente contendo as siglas BR (patente brasileira), PI (Patente de Invenção), PP (Pedido de Patente) e C1 (Certificação de Adição) (Figura 6).

Figura 6. Quantidade de pedidos de preparações médicas por classificação.

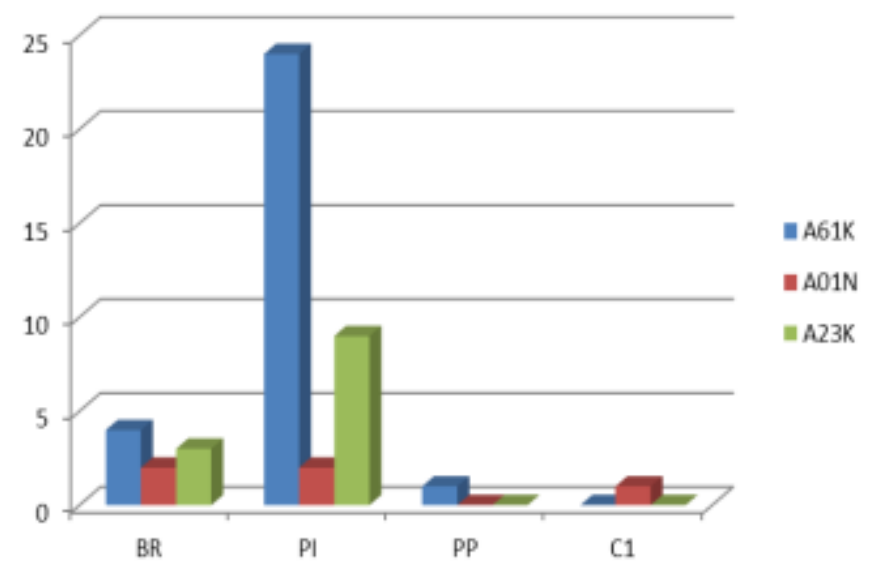

Fonte: Autores.

A classificação A61K é relacionada às preparações para finalidades médicas, odontológicas ou higiênicas, já a 
classificação A01N relaciona a conservação de corpos de seres humanos ou animais ou plantas ou partes dos mesmos. A classificação A23K remete aos produtos alimentícios especialmente adaptados para animais; métodos especialmente adaptados para a produção dos mesmos.

Sendo assim, pode-se observar a grande relevância no depósito de patentes de invenção com finalidades médicas, seguidas dos produtos designados à alimentação e adaptação dos animais e por último, a conservação de tecidos humano, animal ou vegetal.

\section{Conclusão}

Os países europeus são grandes produtores e consumidores de carne de caprinos e os que tem a maior quantidade de tecnologias aplicadas aos manejos destes. Isso fica evidenciado na quantidade de patentes requeridas nos sites internacionais Google Patents e Espacenet.

No Brasil, o estado detentor de grande quantidade de tecnologias agrícolas é São Paulo, estado este com uma quantidade de quase $35 \%$ dos depósitos de patentes que relacionam caprinos.

A tecnificação na área médica é enfatizada com quantidade de patentes brasileiras neste setor, sendo destinadas tanto para melhoramento animal quanto humano.

\section{Referências}

Amparo, K. K. S., Ribeiro, M. C. O., \& Guarieiro, L. L. N. (2012). Estudo de caso utilizando mapeamento de prospecção tecnológica como principal ferramenta de busca científica. Perspectivas em Ciência da Informação, 17(4), 195-209.

Barbosa Filho, J. A., Silva, Y. L., \& Silva, M. C. (2013). Bem-estar no pré-abate é importante no manejo. A Lavoura ${ }^{\circ} 694$.

Barros, J. R. L., \& Cruz, G. R. B. (2017). A produção de caprinos e ovinos na microrregião do cariri ocidental do estado da Paraíba - gargalos e estratégias de intervenção. II CONIDIS.

Campos, F. S., Gois, G. C., Vicente, S. L. A., Macedo, A., \& Matias, A. G. S. (2017). Alternativa de forragem para caprinos e ovinos criados no semiárido. Nutri Time, 14(02).

Cruz, G. R. B., Barros, J. L. R., Santos, D. G., Lima, A. M., \& Silva, A. C. R. (2019). Aspectos sanitários na produção de caprinos e ovinos de produtores familiares no semi-árido paraibano. Revista Conexão UEPG, 15(2).

Feitosa, B. F., Feitoza, J. V. F., Araújo, J. S. F., Xavier, L. E., Soares, W. K. A., \& Cavalcanti, M. T. (2021). Prospecções Científica e Tecnológica Aplicadas a Queijos Caprinos. Cadernos de Prospecção-Salvador, 14(2), 573-58.

Espacenet. https://worldwide.espacenet.com/.

Google Patents. https://patents.google.com/.

INPI - (Instituto Nacional de Propriedade Industrial). http://www.inpi.gov.br/.

Medeiros, A. D., \& Bezerra, J. J. L. (2016). Os desafios para a produção de caprinos no semiárido paraibano. Conidis, I congresso internacional da diversidade do semiárido. v. 1.

Oliveira, L. S. (2020). Características e sustentabilidade de sistemas de produção de caprinos leiteiros no nordeste do Brasil. Tese (Doutorado em Zootecnia), Faculdade de Ciências Agrárias e Veterinárias - UNESP, Campus Jabuticabal.

Silva, C. R., Silva, N. C. S., Costa-Junior, L. M., \& Nunes, G. S. (2019). Avanços no desenvolvimento de tecnologias utilizando óleos essenciais para controle de parasitos de pequenos ruminantes. Revista GEINTEC. 9(2), 4966-4976.

Silva, I. W. H. (2019). Avaliação produtiva, reprodutiva e econômica de caprinos leiteiros no semiárido. Dissertação (Mestrado em Zootecnia), Universidade Federal de Campina Grande.

Vaz, V. H. S. (2019). Avaliação do depósito de patentes no Brasil a partir dos dispêndios públicos em ciência e tecnologia. PIDCC, Aracaju/Se, Ano VIII, 13(03), 084-092. 